\title{
Differentially expressed long non-coding RNAs and mRNAs in patients with IgA nephropathy
}

\author{
NAN ZUO ${ }^{1}$, YUN LI $^{2}$, NAN LIU ${ }^{1}$ and LINING WANG ${ }^{1}$ \\ ${ }^{1}$ Division of Nephrology, The First Affiliated Hospital of China Medical University, Shenyang, Liaoning 110001; \\ ${ }^{2}$ Division of Nephrology, The People's Hospital of Tacheng, Tacheng, Xinjiang 834700, P.R. China
}

Received December 1, 2016; Accepted July 5, 2017

DOI: $10.3892 / \mathrm{mmr} .2017 .7542$

\begin{abstract}
Long non-coding RNAs (lncRNAs) have been reported to serve a crucial role in renal diseases; however, their role in immunoglobulin A nephropathy (IgAN) remains unclear. In the present study, peripheral blood mononuclear cells (PBMCs) were collected from both patients with IgAN and healthy controls. A microarray analysis was then performed to identify differentially expressed lncRNAs and mRNAs in PBMCs, which were confirmed by quantitative polymerase chain reaction. In addition, Gene Ontology (GO), Kyoto Encyclopedia of Genes and Genomes (KEGG) pathway and lncRNA-mRNA co-expression network analyses were conducted. The present study identified 167 differentially expressed lncRNAs and 94 differentially expressed mRNAs. Numerous GO terms, including innate immune response, inflammatory response, IPAF inflammasome complex and UDP-galactose: $\beta-\mathrm{N}$-acetylglucosamine $\beta$ - 1 , and 3 -galactosyltransferase activity, were significantly enriched in the differentially expressed mRNAs. The top five KEGG signaling pathways included nucleotide-binding oligomerization domain-like receptor signaling pathway, hematopoietic cell lineage, inflammatory bowel disease, tumor necrosis factor signaling pathway and other types of O-glycan biosynthesis. In addition, a total of 149 lncRNAs were shown to interact with 7 mRNAs that were associated with the innate immune response' GO term. The results of the present study demonstrated that differentially expressed lncRNAs and mRNAs may have a role in the development of IgAN. These results may aid in the elucidation of a basic pathogenic mechanism, the identification of possible biomarkers and the generation of potential novel treatment strategies for $\operatorname{IgAN}$.
\end{abstract}

Correspondence to: Dr Lining Wang, Division of Nephrology, The First Affiliated Hospital of China Medical University, 155 Nanjing North Street, Shenyang, Liaoning 110001, P.R. China

E-mail: lnwang56@medmail.com.cn

Key words: immunoglobulin A nephropathy, long non-coding RNA, mRNA, innate immunity response, co-expression network

\section{Introduction}

Immunoglobulin ( $\operatorname{Ig})$ A nephropathy $(\operatorname{IgAN})$ is considered the leading cause of primary glomerulonephritis worldwide (1). Mesangial hypercellularity and matrix expansion, alongside glomerular IgA deposition, which according to renal biopsies is usually accompanied by complement component 3 and $\mathrm{IgG}$ deposition, are all involved in its pathogenesis (1). Approximately $40 \%$ of patients with $\operatorname{Ig}$ AN will develop end-stage renal disease (ESRD) within 20 years of the initial biopsy (2). IgAN is a complex multifactorial disease associated with various clinical and pathological features, and its pathogenic mechanisms remain unknown.

Non-coding RNAs (ncRNAs) were once considered to have no significant transcriptional functions; however, they have been increasingly reported to serve an important role in cell differentiation and disease progression (3). Long ncRNAs (lncRNAs) are a class of transcripts $>200$ nucleotides in length with little or no protein-coding capacity $(4,5)$. However, lncRNAs do participate in various biological processes, including gene expression, recruitment of chromatin modifications, $\mathrm{X}$ chromosome inactivation, chromosome recombination and protein folding $(6,7)$. Recent studies regarding lncRNAs in kidney diseases have reported that differentially expressed lncRNAs are present in ESRD (8) and IgA-negative mesangial proliferative glomerulonephritis (9). In addition, some lncRNAs may be used as biomarkers in membrane nephropathy (10) and diabetic nephropathy (11). Although the detailed regulatory mechanisms and functions of IncRNAs remain uncertain, they form a basis for subsequent research on the development of renal diseases.

At present, to the best of our knowledge, the expression of lncRNAs and their influence on IgAN have yet to be reported. The present study aimed to determine the altered expression of lncRNAs and mRNAs profiled in patients with $\operatorname{IgAN}$. Furthermore, via Gene Ontology (GO), Kyoto Encyclopedia of Genes and Genomes (KEGG) pathway and lncRNA-mRNA co-expression network analyses, the study aimed to elucidate the functions of these dysregulated lncRNAs and mRNAs in the pathogenesis and progression of $\operatorname{IgAN}$.

\section{Subjects and methods}

Isolation of peripheral blood mononuclear cells (PBMCs) from patients with IgAN and controls. A total of 12 patients 
with $\operatorname{IgAN}$ and 12 healthy controls were recruited to the present study from the First Affiliated Hospital of China Medical University (Shenyang, China), between November and December 2015. The patients were diagnosed with IgAN according to renal immunopathological diagnosis (by kidney biopsy) and due to the presence of clinical characteristics without other complications. Patients with secondary IgAN were excluded from the present study, and the patients studied had never been treated with immunosuppressive drugs. The present study was approved by the Ethical Review Committees of the First Affiliated Hospital of China Medical University, and were conducted in accordance with the Declaration of Helsinki. Written informed consent was obtained from all participants, and blood samples were collected from patients with IgAN and healthy controls. PBMCs were isolated using Ficoll-Hypaque gradient centrifugation (12).

Microarray analysis. Total RNAs were extracted from PBMCs using TRIzol (Invitrogen; Thermo Fisher Scientific, Inc., Waltham, MA, USA) and were purified with the RNeasy kit (Qiagen $\mathrm{GmbH}$, Hilden, Germany) according to the manufacturers' protocols; individual RNA samples were stored at $-80^{\circ} \mathrm{C}$ until further use. RNA concentration was quantified using the NanoDrop ND-2000 (NanoDrop; Thermo Fisher Scientific, Inc., Wilmington, DE, USA). After passing RNA measurement quality control using the NanoDrop ND-2000 and denaturing gel electrophoresis, RNA was used to synthesize double-stranded cDNA using the SuperScript Double-Stranded cDNA Synthesis kit (Invitrogen; Thermo Fisher Scientific, Inc.) according to manufacturer's protocol.

The Agilent Human lncRNA (4*180K, Design ID, 076500; Agilent Technologies, Inc., Santa Clara, CA, USA) was used to conduct the microarray analysis according to manufacturer's protocol. Differentially expressed mRNAs were also identified using a microarray. Raw data were extracted as pair files by Feature Extraction software (version 10.7.1.1; Agilent Technologies, Inc.), and Genespring (version 13.1; Agilent Technologies, Inc.) was used to analyze the raw data. The threshold set for up- and downregulated genes was a fold change $\geq 2.0$ and $\mathrm{P} \leq 0.05$.

Reverse transcription-quantitative polymerase chain reaction $(R T-q P C R)$ validation. Total RNA was reverse transcribed using Random Decamers (Ambion; Thermo Fisher Scientific, Inc., Waltham, MA, USA) and reverse transcriptase, M-MLV (Invitrogen; Thermo Fisher Scientific, Inc.): cDNA synthesis was $25^{\circ} \mathrm{C}$ for $5 \mathrm{~min}, 42^{\circ} \mathrm{C}$ for $60 \mathrm{~min}$ and $70^{\circ} \mathrm{C}$ for $5 \mathrm{~min}$. lncRNA expression levels were quantified by qPCR using a SYBR-Green kit (Thermo Fisher Scientific, Inc.). Reactions were performed in duplicate and comprised $2 \mathrm{X}$ concentrated Universal Master mix, $1 \mu 1$ template cDNA, and $100 \mathrm{nM}$ primers in a final volume of $9 \mu \mathrm{l}$. Reactions were analyzed in a 384-well PCR reaction plate (Axygen; Corning Incorporated, Corning, NY, USA) using a QuantStudio ${ }^{\mathrm{TM}} 6$ Flex Real-Time PCR system (Applied Biosystems; Thermo Fisher Scientific, Inc., Waltham, MA, USA). The RT-qPCR conditions were as follows: PCR amplification for 40 cycles at $95^{\circ} \mathrm{C}$ for $10 \mathrm{sec}$, at $60^{\circ} \mathrm{C}$ for $60 \mathrm{sec}$ and at $95^{\circ} \mathrm{C}$ for $10 \mathrm{sec}$. Then, the temperature was allowed to slowly rise from 60 to $99^{\circ} \mathrm{C}$. The relative lncRNA expression levels were calculated using the
Table I. Characteristics of patients with $\operatorname{IgAN}$ and healthy controls enrolled in the present study.

\begin{tabular}{lcc}
\hline Characteristic & IgAN & Controls \\
\hline Age, mean \pm SD (years) & $35 \pm 12$ & $36 \pm 10$ \\
Sex (M:F) & $7: 5$ & $1: 1$ \\
Urine RBCs count & $115(40-240)$ & 0 \\
(range, $\left.10^{4} / \mathrm{ml}\right)$ & & \\
Proteinuria $(\mathrm{g} /$ day $)$ & $0.87 \pm 0.50$ & $0.15 \pm 0.10$ \\
Scr, mean \pm SD $(\mu \mathrm{mol} / \mathrm{l})$ & $151.50 \pm 90.20$ & $58.50 \pm 21.50$
\end{tabular}

F, female; IgAN, immunoglobulin A nephropathy; M, male; RBCs, red blood cells; Scr, serum creatinine.

$\Delta \Delta \mathrm{C}_{\mathrm{q}}$ method (13) and data were normalized with $\mathrm{H}$-actin. Primer sequences were as follows: Myelin expression factor 2 (MYEF2)-1:1 forward, 5'-CCCAACACAAGCTAGAAG ACAACA-3' and reverse, 5'-GCATCTTGCTACTTTAAT TGGTCC-3'; MYEF2-1:3 forward, 5'-TACACACATGCT TACATTTTGAAGG-3' and reverse, 5'-GCACCAACTAAT ACCAAGGAACC-3'; MYEF2-1:4 forward, 5'-TTCCCCAAT ACGTTAATGTTTTGAC-3' and reverse, 5'-GGAAAAAGG AGGAGTGAATGGGT-3'; arachidonate 15-lipoxygenase pseudogene 1 (ALOX15P1)-nc forward, 5'-TGTTGACTT TAAGGTTTCGCTGG-3' and reverse, 5'-CAGAGCGCCTCA GCACCAC-3'; mitogen-activated protein kinase 8 interacting protein 2 (MAPK8IP2)-1:14 forward, 5'-CAAGCCAGAGCG GGTCAGTT-3' and reverse, 5'-GGGCACTGGGAGAAG TTAGCAC-3'; folliculogenesis specific BHLH transcription factor (FIGLA)-1:1 forward, 5'-GCCGACCGGAGATAGCTA AGA-3' and reverse, 5'-GGCTCCTTCTGTTCACTGCTCA-3'; and $\mathrm{H}$-actin forward, 5-AGCACAGAGCCTCGCCTTTG-3' and reverse, 5'-CTTCTGACCCATGCCCACCA-3'.

GO analysis and KEGG pathway. GO is an ontological classification analysis ascribing functions of differentially expressed mRNAs to GO categories. Classification consists of cellular components, molecular functions and biological processes. The GO categories are derived from the GO Consortium (www.geneontology.org), which comprises three structured networks of defined terms used to describe the gene product attributes. KEGG (http://www.genome.jp/kegg/pathway. $\mathrm{html} /$ ) mapping can be used to predict which biological pathways differentially expressed mRNAs are involved in. According to P-value, significantly enriched GO terms and KEGG pathways were screened. $\mathrm{P} \leq 0.05$ was considered to indicate significance.

lncRNA-mRNA co-expression network. The IncRNA-mRNA co-expression network was based on a correlation between differentially expressed lncRNAs and mRNAs enriched in the 'innate immune response' term, and was constructed according to normalized signal intensities of specific expression levels of lncRNAs and mRNAs. Pearson's correlation coefficients $(>0.9)$ were used to identify the lncRNAs and associated coding genes. Subsequently, the IncRNA-mRNA co-expression network was constructed using Cytoscape 
Table II. Top 20 differentially expressed lncRNAs.

\begin{tabular}{ll}
\hline Order & \multicolumn{1}{c}{ lncRNA } \\
\hline 1 & ENST00000496886 \\
2 & DNAJC24-3:1 \\
3 & TMEM63B-1:1 \\
4 & FAM27L-7:1 \\
5 & MYEF2-1:1 \\
6 & TMEM100-3:1 \\
7 & NRG2-1:6 \\
8 & DNAJA1-3:1 \\
9 & PABPC3-3:1 \\
10 & PARP16-4:1 \\
11 & ZNF296-2:4 \\
12 & ENST00000478301 \\
13 & WHAMM-2:2 \\
14 & SAP30-2:1 \\
15 & SOD1-9:1 \\
16 & ENST00000448680 \\
17 & CDYL2-2:10 \\
18 & ENST00000564702 \\
19 & FBN1-3:2 \\
20 & CYBRD1-3:1 \\
\hline
\end{tabular}

lncRNA, long non-coding RNA.

Table III. Top 20 differentially expressed mRNAs.

\begin{aligned} \hline Order & \multicolumn{1}{c}{ mRNA } \\ \hline 1 & SLC12A1 \\ 2 & TCONS_12_00008986 \\ 3 & KIAA1324 \\ 4 & TCONS_00019298 \\ 5 & SLC12A1 \\ 6 & ZFP57 \\ 7 & GUCY1A3 \\ 8 & OLIG2 \\ 9 & PGA3 \\ 10 & IL5RA \\ 11 & IL18RAP \\ 12 & CLEC7A \\ 13 & TCONS_12_00008982 \\ 14 & TCONS_00021505 \\ 15 & GOLGA6L6 \\ 16 & ALOX15 \\ 17 & PRSS33 \\ 18 & SIGLEC12 \\ 19 & LOC100653133 \\ 20 & DEFB123 \\ \hline & \end{aligned}

software version 2.8.1 (The Cytoscape Consortium, San Diego, CA, USA).

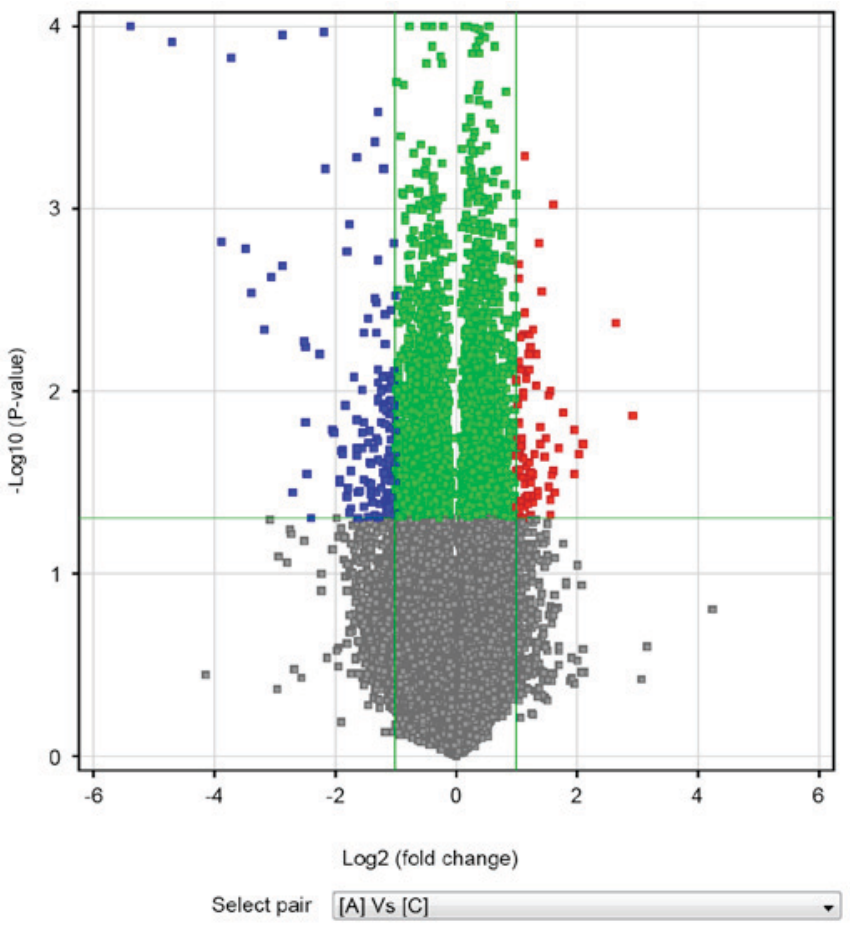

Figure 1. Volcano plot of lncRNAs. The grey and green spots indicate non-significant differentially expressed lncRNAs (fold change $<2.0$ and $\mathrm{P}>0.05$ ); red spots indicate significantly upregulated lncRNAs; and blue spots indicate significantly downregulated lncRNAs compared with the control group (fold change $\geq 2.0$ and $\mathrm{P} \leq 0.05$ ). IncRNA, long non-coding RNA.

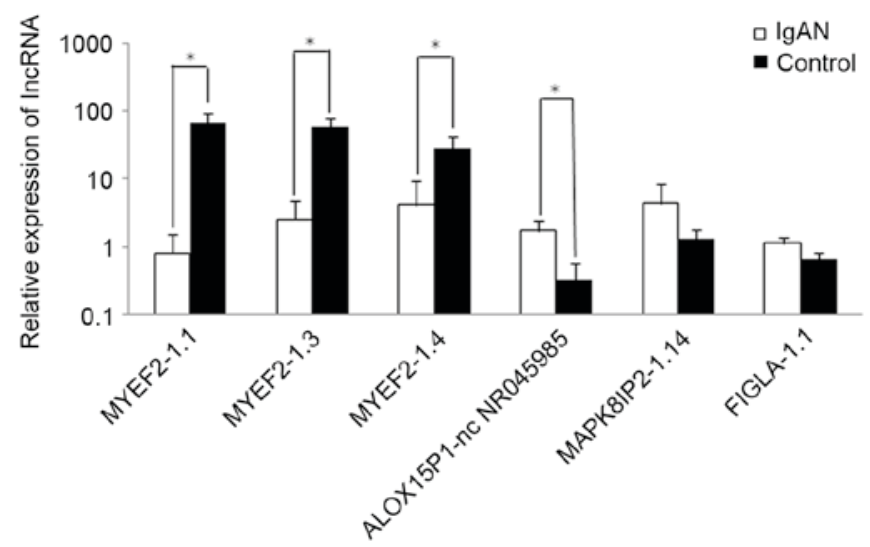

Figure 2. RT-qPCR verification of lncRNA microarray data. Three upregulated and three downregulated lncRNAs were validated by RT-qPCR analysis of RNA extracted from the peripheral blood mononuclear cells of 12 patients with IgAN and 12 healthy controls. The relative lncRNA expression levels were normalized to $\mathrm{H}$-actin, and data are presented as the mean \pm standard deviation. "P<0.05. ALOX15P1, arachidonate 15-lipoxygenase pseudogene 1; FIGLA, folliculogenesis specific BHLH transcription factor; IgAN, immunoglobulin A nephropathy; lncRNA, long non-coding RNA; MAPK8IP2, mitogen-activated protein kinase 8 interacting protein 2; MYEF, myelin expression factor 2; RT-qPCR, reverse transcription-quantitative polymerase chain reaction.

Statistical analysis. Data are presented as the mean \pm standard deviation. Data obtained from three independent repetitions of RT-qPCR were analyzed using unpaired Student's t-test. Statistical analyses were performed using SPSS software version 18.0 (SPSS, Inc., Chicago, IL, USA). P<0.05 was considered to indicate a statistically significant difference. 


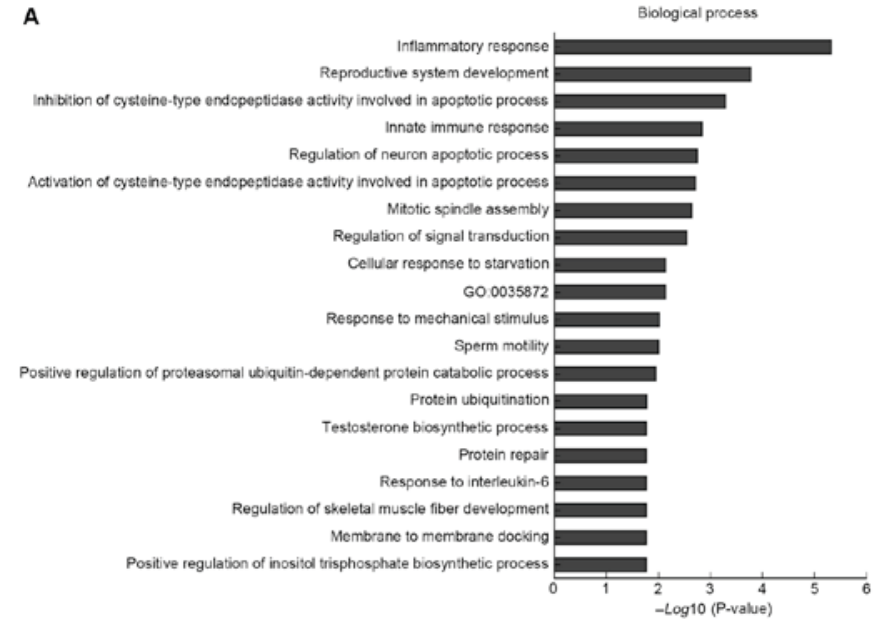

B
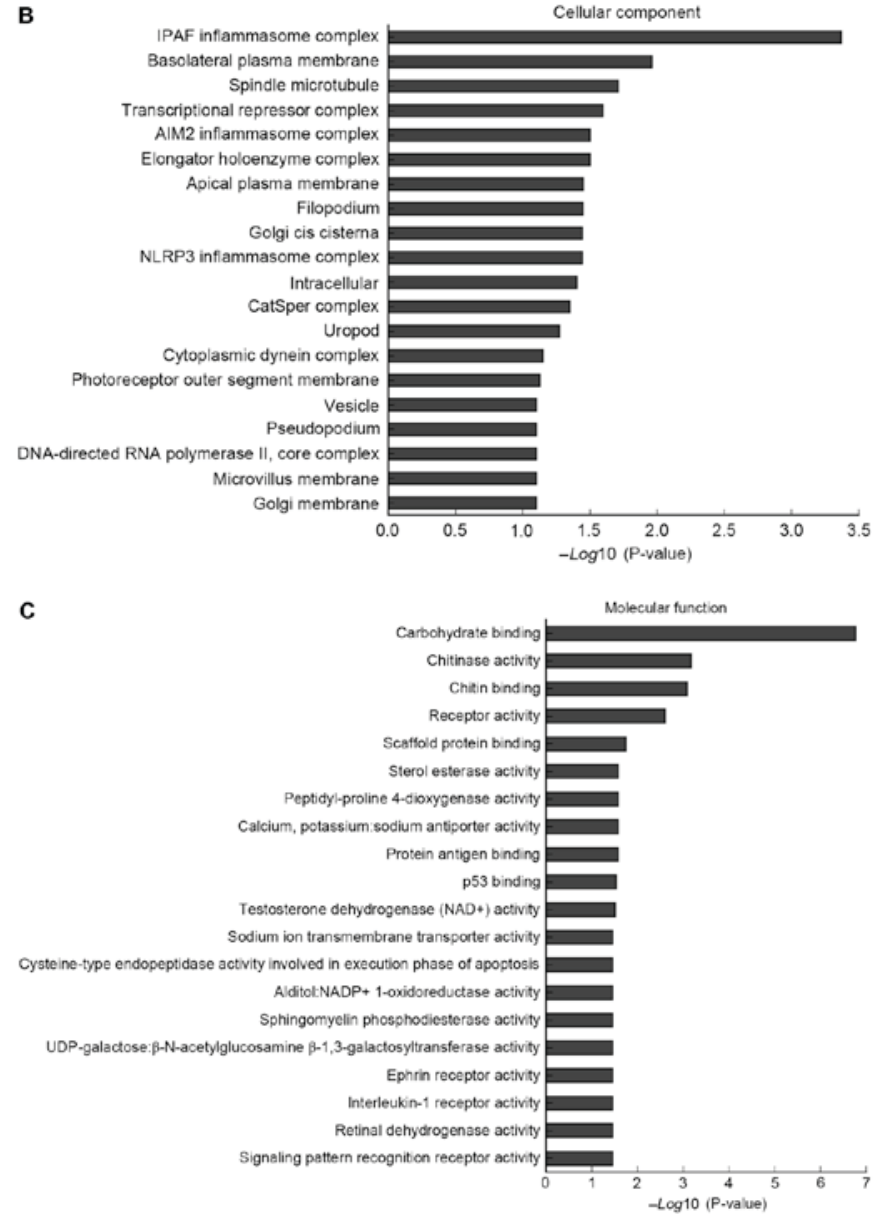

Figure 3. GO analysis for differentially expressed mRNAs. Graphs represent the enrichment of these mRNAs. The - $\log 10$ (P-value) was a positive correlation with GO. (A) Biological process; (B) cellular component; and (C) molecular function. GO, Gene Ontology.

\section{Results}

Differentially expressed lncRNAs and mRNAs in IgAN. The demographics and baseline clinical characteristics of the study subjects are summarized in Table I. lncRNA and mRNA expression in PBMCs from 12 patients with IgAN and 12 healthy controls were studied using microarray analyses. A total of 167 differentially expressed lncRNAs (including 55 upregulated lncRNAs and 112 downregulated lncRNAs)

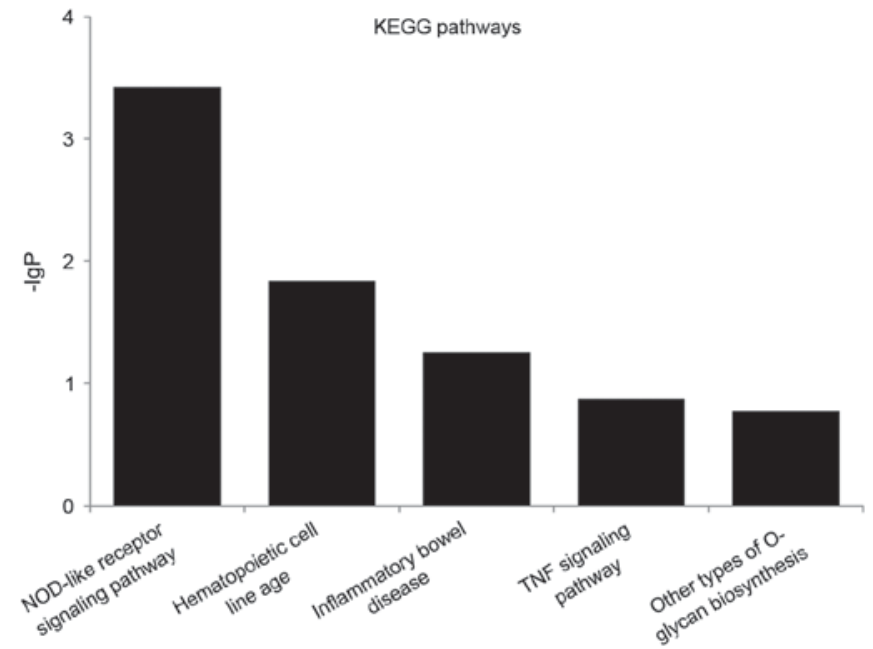

Figure 4. KEGG pathways for differentially expressed mRNAs. The graph represents the enrichment of these mRNAs. The top five significantly enriched KEGG pathways were calculated and plotted as -Log10 (P-value). KEGG, Kyoto Encyclopedia of Genes and Genomes; NOD, nucleotide-binding oligomerization domain; TNF, tumor necrosis factor.

and 94 differentially expressed mRNAs (including 36 upregulated mRNAs and 58 downregulated mRNAs) were identified. The differentially expressed lncRNAs varied between the patients with IgAN and the healthy controls, as shown by a volcano plot (Fig. 1). The red and the blue spots indicate increased or decreased expression compared with relative expression, respectively. The top 20 expressed lncRNAs and mRNAs are listed in Tables II and III.

$R T-q P C R$ validation. For the purpose of validating the microarray analysis results and determining the role of lncRNAs in IgAN, 6 lncRNAs were randomly selected. As presented in Fig. 2, differential expression of these lncRNAs was detected in patients with $\operatorname{IgAN}$ compared with healthy controls. IncRNA MYEF2-1.1, lncRNA MYEF2-1.3 and lncRNA MYEF2-1.4 exhibited 85-, 22.92- and 6.91-fold lower expression in patients with IgAN, respectively. In addition, lncRNA ALOX15P1-nc NR045985, lncRNA MAPK8IP2-1.14 and lncRNA FIGLA-1.1 exhibited 5.15-, 3.38- and 1.68-fold higher expression in patients with $\operatorname{IgAN}$, respectively. These results agreed with the findings obtained from the microarray analysis.

GO and KEGG analyses of differentially expressed mRNAs. A total of 94 filtered mRNAs were included in the GO analysis. As shown in Fig. 3. GO analysis of the differentially expressed mRNAs indicated that they were enriched in numerous GO terms, including innate immune response, inflammatory response, IPAF inflammasome complex and UDP-galactose: $\beta$-N-acetylglucosamine $\beta$-1, and 3 -galactosyltransferase activity.

Furthermore, the genes were also mapped to pathways in a functionally analytical manner; the top five KEGG pathways are listed in Fig. 4, including nucleotide-binding oligomerization domain (NOD)-like receptor signaling pathway, hematopoietic cell lineage, inflammatory bowel disease (IBD), tumor necrosis factor (TNF) signaling pathway and other types of O-glycan biosynthesis. 


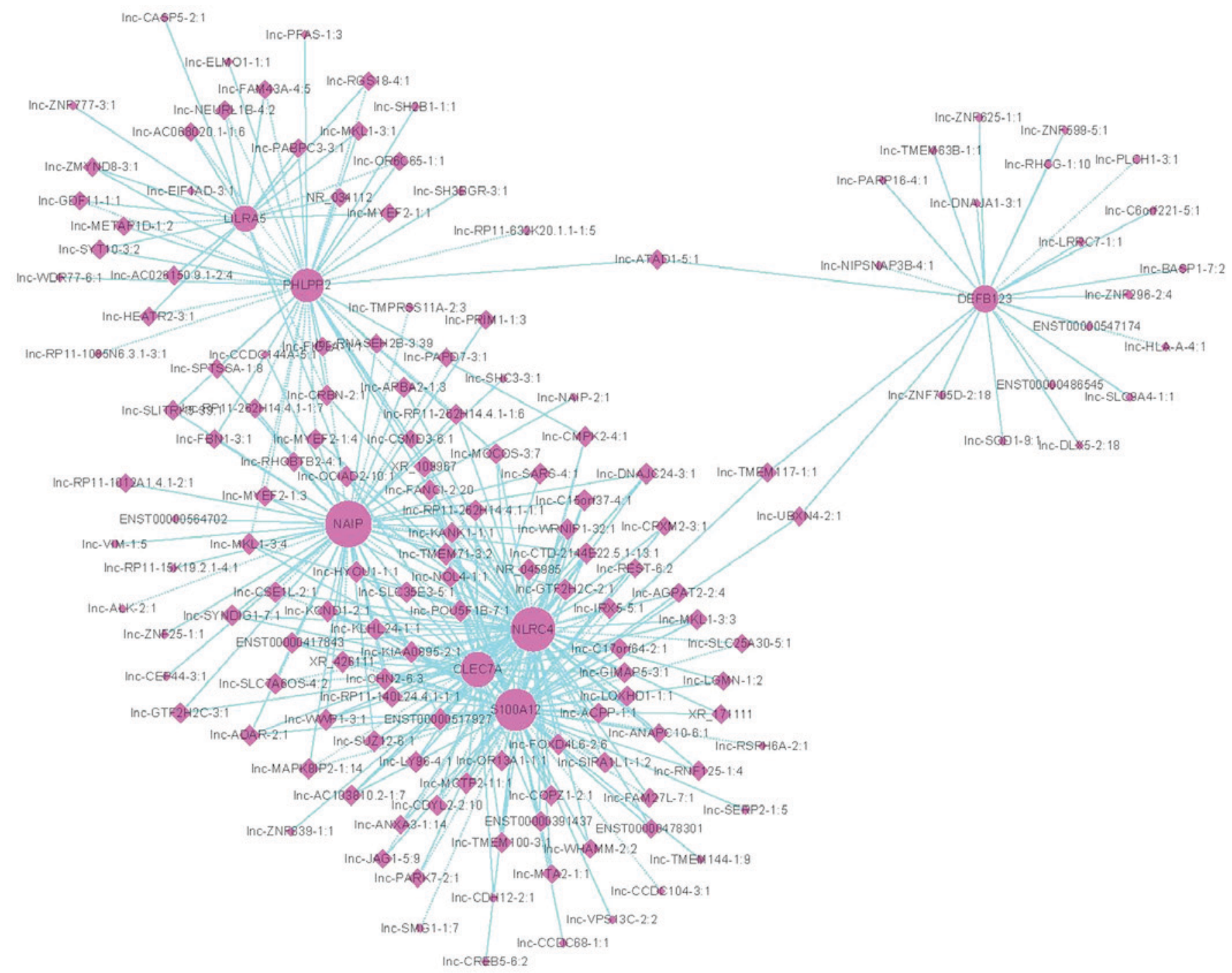

Figure 5. IncRNA-mRNA co-expression network. A total of 149 lncRNAs interacted with 7 mRNAs that were associated with the 'innate immune response' Gene Ontology term. lncRNA, long non-coding RNA.

IncRNA-mRNA co-expression network. In the enriched biological processes identified in the GO analysis, the term 'innate immune response' was analyzed further, in order to generate a lncRNA-mRNA co-expression network. A total of 167 differentially expressed lncRNAs and 94 mRNAs comprised the network analysis; 149 lncRNAs were revealed to interact with 7 mRNAs participating in this term (Fig. 5).

\section{Discussion}

IgAN represents a common form of primary glomerulonephritis, which is characterized by highly heterogeneous clinicopathological features (1), and is an important cause of ESRD. Approximately half of patients with IgAN will eventually depend on dialysis. While the pathogenesis of $\operatorname{IgAN}$ is only partially understood, previous studies support a multi-hit hypothesis: Specific autoantibodies recognize galactose-deficient $\operatorname{Ig} \mathrm{A}_{1}$ molecules, resulting in the formation of circulating IgA1-IgG immune complexes, which may be deposited in the glomerular mesangium, thereby inducing renal injury $(1,14)$.

Regulatory ncRNAs have essential roles in the regulation of gene expression and mammalian development, and include
microRNAs (miRNAs/miRs) and lncRNAs $(15,16)$. miRNAs have been reported to contribute to the progression of IgAN and may potentially serve as biomarkers for diagnosis and disease monitoring (17); for example, abnormal expression of miR-148b may explain aberrant glycosylation of $\operatorname{Ig} \mathrm{A}_{1}$ (18). Furthermore, a retrospective international study indicated that circulating miR-148b and let-7b may be considered serum markers for detecting primary IgAN (19). However, research regarding the role of lncRNAs in IgAN is lacking. The establishment of differentially expressed lncRNA profiles in human IgAN is important for elucidating an explicit pathogenesis of this illness.

The present study generated IncRNA and mRNA expression profiles in PBMCs from patients with IgAN and healthy controls via microarray analyses. A total of 167 differentially expressed lncRNAs and 94 differentially expressed mRNAs were identified, thus suggesting that these mRNAs and lncRNAs may serve as potential biomarkers for the diagnosis of IgAN. Via GO and KEGG pathway analyses, detailed information was obtained regarding the biological functions and potential mechanisms of these mRNAs in IgAN. Numerous GO terms, including innate immune response 
and inflammatory response, were revealed to be significantly enriched in the identified differentially expressed genes. In addition, 94 differentially expressed mRNAs were associated with numerous KEGG pathways; the top five pathways included NOD-like receptor signaling pathway, hematopoietic cell lineage and IBD.

The present study focused on the 'innate immune response' term in $\mathrm{GO}$ analysis when evaluating the prominent role of innate immune dysregulation in IgAN. Toll-like receptors (TLR) are a family of pathogen recognition molecules, including TLR1, TLR3, TLR4, TLR9 and TLR10, which have been reported to be associated with the development of $\operatorname{IgAN}$. A nasal challenge with $\mathrm{CpG}$ oligodeoxynucleotide, which is a ligand of TLR9, was reported to aggravate renal injury in a murine IgAN model (20), and a single nucleotide polymorphism in TLR9 (TT genotype in rs352140) was reported to be an important risk factor for the progression of human IgAN (19). In addition, TLR9 on B cells and dendritic cells located in the mucosa may serve different roles in the development of $\operatorname{IgAN}$, via induction of nephritogenic IgA or IgA-IgG immune complexes, respectively (21). Such findings suggested that TLR9 may be closely associated with the pathogenesis of human IgAN. Coppo et al demonstrated that the levels of TLR4 in the PBMC of patients with IgAN were increased, and were correlated with the degree of proteinuria and gross microscopic hematuria (22). Furthermore, TLR1 (CT and CC genotype in rs4833095, TT genotype in rs5743557) (23) and TLR10 genes (TA and AA genotype in rs1004195) (24) may be associated with susceptibility to IgAN in Korean children. Recently, He et al (25) reported that the TLR3-B-cell activating factor axis was involved in IgA class switch recombination in IgAN.

Activation of the complement system, including the alternative and lectin pathways, has also been reported to be closely associated with IgAN, perhaps by contributing to abnormal IgA1 glycosylation $(26,27)$. Furthermore, local renal polarization of macrophages has been implicated in the pathological type of $\operatorname{Ig} \mathrm{AN}$; activation of M2 macrophages was followed by fibrotic alterations, whereas M1 polarization induced mesangial proliferation (21).

Existing evidence has indicated that innate immune disturbances crucially contribute to the pathogenesis of $\operatorname{IgAN}$; however, these require further investigation. The present results obtained from the $\mathrm{GO}$ analysis in patients with $\mathrm{IgAN}$ supported the importance of the 'innate immune response', which is concordant with the findings of previous studies. Therefore, the present study focused on the innate immune response' term for further lncRNA-mRNA co-expression network analysis. The co-expression network was constructed based on the 167 differentially expressed lncRNAs and the 94 differentially expressed mRNAs obtained from patients with IgAN compared with healthy controls. A total of 149 lncRNAs were revealed to interact with 7 mRNAs that participated in the 'innate immune response' term. These mRNAs included NAIP, LILRA5 and CLEC7A. The protein encoded by LILRA5 is a member of the leukocyte immunoglobulin-like receptor (LIR) family, known to have activating and inhibitory functions in leukocytes. There are 19 lncRNAs interacting with LILRA5 in the 'innate immune response' term. Lnc-CRBN-2:1 is one of these lncRNAs and is also known as IL5RA. The protein encoded by this gene is an interleukin 5 specific subunit of a heterodimeric cytokine receptor. A potential interaction of C1GALT1 and IL5RA on the susceptibility of IgAN has been identified (28). These findings suggested that the inter-regulation of lncRNAs and mRNAs may be involved in IgAN-associated innate immune disturbances. Dysregulation in the lncRNA-mRNA network may be a possible mechanism underlying IgAN progression.

In conclusion, the present study systematically screened abnormally expressed lncRNAs and mRNAs between patients with IgAN and healthy controls, providing novel information regarding the potential role of lncRNAs in IgAN. GO and KEGG pathway analyses revealed detailed information regarding the biological functions and potential mechanisms of these mRNAs. In addition, the co-expression network generated in the present study suggested inter-regulation of lncRNAs and mRNAs in patients with IgAN. These findings form a basis for further studies of lncRNAs in IgAN, which should focus on exploring the functions and regulatory mechanisms of identified lncRNAs, thus identifying potential screening biomarkers and novel treatment targets for this disease.

\section{References}

1. Wyatt RJ and Julian BA: IgA nephropathy. N Engl J Med 368: 2402-2414, 2013

2. Schena FP: A retrospective analysis of the natural history of primary IgA nephropathy worldwide. Am J Med 89: 209-215, 1990.

3. Consortium EP: An integrated encyclopedia of DNA elements in the human genome. Nature 489: 57-74, 2012.

4. Cheetham SW, Gruhl F, Mattick JS and Dinger ME: Long noncoding RNAs and the genetics of cancer. Br J Cancer 108: 2419-2425, 2013

5. Ma L, Bajic VB and Zhang Z: On the classification of long non-coding RNAs. RNA Biol 10: 925-933, 2013.

6. Fatica A and Bozzoni I: Long non-coding RNAs: New players in cell differentiation and development. Nat Rev Genet 15: 7-21, 2014.

7. Wang KC and Chang HY: Molecular mechanisms of long noncoding RNAs. Mol Cell 43: 904-914, 2011.

8. Sui W, Yan Q, Li H, Liu J, Chen J, Li L and Dai Y: Genome-wide analysis of long noncoding RNA expression in peripheral blood monoclear cells of uremia patients. J Nephrol 26: 731-738, 2013.

9. Sui W, Li H, Ou M, Tang D and Dai Y: Altered long non-coding RNA expression profile in patients with IgA-negtive mesangial proliferative glomerulonephritis. Int J Mol Med 30: 173-178, 2012.

10. Huang YS, Hsieh HY, Shih HM, Sytwu HK and Wu CC: Urinary Xist is a potential biomarker for membranous nephropathy. Biochem Biophys Res Commun 452: 415-421, 2014.

11. Alvarez ML, Khosroheidari M, Eddy E and Kiefer J: Role of microRNA $1207-5 \mathrm{P}$ and its host gene, the long non-coding RNA Pvt1, as mediators of extracellular matrix accumulation in the kidney: Implications for diabetic nephropathy. PLoS One 8: e77468, 2013.

12. Wang J, Peng H, Tian J, Ma J, Tang X, Rui K, Tian X, Wang Y, Chen J, Lu L, et al: Upregulation of long noncoding RNA TMEVPG1 enhances T helper type 1 cell response in patients with Sjögren syndrome. Immunol Res 64: 489-496, 2016.

13. Livak KJ and Schmittgen TD: Analysis of relative gene expression data using real-time quantitative PCR and the 2(-Delta Delta C(T)) method. Methods 25: 402-408, 2001.

14. Novak J, Renfrow MB, Gharavi AG and Julian BA: Pathogenesis of immunoglobulin A nephropathy. Curr Opin Nephrol Hypertens 22: 287-294, 2013.

15. Ponting CP, Oliver PL and Reik W: Evolution and functions of long noncoding RNAs. Cell 136: 629-641, 2009.

16. Zaratiegui M, Irvine DV and Martienssen RA: Noncoding RNAs and gene silencing. Cell 128: 763-776, 2007.

17. Szeto CC and Li PK: MicroRNAs in IgA nephropathy. Nat Rev Nephrol 10: 249-256, 2014. 
18. Serino G, Sallustio F, Cox SN, Pesce F and Schena FP: Abnormal miR-148b expression promotes aberrant glycosylation of IgA1 in IgA nephropathy. J Am Soc Nephrol 23: 814-824, 2012.

19. Serino G, Pesce F, Sallustio F, De Palma G, Cox SN, Curci C, Zaza G, Lai KN, Leung JC, Tang SC, et al: In a retrospective international study, circulating miR-148b and let-7b were found to be serum markers for detecting primary IgA nephropathy. Kidney Int 89: 683-692, 2016.

20. Suzuki H, Suzuki Y, Narita I, Aizawa M, Kihara M, Yamanaka T, Kanou T, Tsukaguchi H, Novak J, Horikoshi S and Tomino Y: Toll-like receptor 9 affects severity of IgA nephropathy. J Am Soc Nephrol 19: 2384-2395, 2008.

21. Kajiyama T, Suzuki Y, Kihara M, Suzuki H, Horikoshi S and Tomino Y: Different pathological roles of toll-like receptor 9 on mucosal B cells and dendritic cells in murine IgA nephropathy. Clin Dev Immunol 2011: 819646, 2011.

22. Coppo R, Camilla R, Amore A, Peruzzi L, Daprà V, Loiacono E, Vatrano S, Rollino C, Sepe V, Rampino T and Dal Canton A: Toll-like receptor 4 expression is increased in circulating mononuclear cells of patients with immunoglobulin A nephropathy. Clin Exp Immunol 159: 73-81, 2010.

23. Lee JS, Park HK, Suh JS, Hahn WH, Kang SW, Park HJ, Kim MJ, Chung JH and Cho BS: Toll-like receptor 1 gene polymorphisms in childhood IgA nephropathy: A case-control study in the Korean population. Int J Immunogenet 38: 133-138, 2011.
24. Park HJ, Hahn WH, Suh JS, Kim MJ, Kang SW, Lee JS, Kim JW, Chung JH and Cho BS: Association between toll like receptor 10 (TLR10) gene polymorphisms and childhood IgA nephropathy. Eur J Pediatr 170: 503-509, 2011.

25. He L, Peng X, Wang J, Tang C, Zhou X, Liu H, Liu F, Sun L and Peng Y: Synthetic double-stranded RNA Poly(I:C) aggravates IgA nephropathy by triggering IgA class switching recombination through the TLR3-BAFF axis. Am J Nephrol 42: 185-197, 2015.

26. Hashimoto A, Suzuki Y, Suzuki H, Ohsawa I, Brown R, Hall S, Tanaka Y, Novak J, Ohi H and Tomino Y: Determination of severity of murine IgA nephropathy by glomerular complement activation by aberrantly glycosylated $\operatorname{Ig} \mathrm{A}$ and immune complexes. Am J Pathol 181: 1338-1347, 2012.

27. Karsten CM, Pandey MK, Figge J, Kilchenstein R, Taylor PR Rosas M, McDonald JU, Orr SJ, Berger M, Petzold D, et al: Anti-inflammatory activity of IgG1 mediated by Fc galactosylation and association of FcgammaRIIB and dectin-1. Nat Med 18: 1401-1406, 2012.

28. Wang W, Sun Y, Fu Y, Yu X and Li M: Interaction of C1GALT1-IL5RA on the susceptibility to IgA nephropathy in Southern Han Chinese. J Hum Genet 58: 40-46, 2013. 